\title{
Decision Support for Adopting SPLE with Transit-PL
}

\author{
Mert Emin Kalender \\ Bilkent University \\ Dept. of Computer \\ Engineering \\ 06800 Bilkent, Ankara, Turkey \\ mert.kalender@bilkent.edu.tr \\ R\&D Department
06800 Ankara, Turkey \\ etuzun@havelsan.com.tr
}

\author{
Bedir Tekinerdogan \\ Bilkent University \\ Dept. of Computer \\ Engineering \\ 06800 Bilkent, Ankara, Turkey \\ bedir@bilkent.edu.tr
}

\begin{abstract}
It is generally acknowledged that the decision to adopt a software product line engineering (SPLE) approach needs to be performed carefully due to the different risks involved in taking such an important decision. To mitigate the potential risks of the transition to SPLE, several studies have been proposed that include many different rules for analyzing the feasibility of the SPLE adoption and the selection of transition process. However, it is not easy to apply these manually and likewise provide a proper decision with the corresponding justification. In this paper, we propose the tool Transit-PL, a web based decision support system for analyzing the feasibility of SPLE for an organization and selecting the appropriate transition strategy. Transit-PL provides a framework to build particular decision support system for selected strategies using different types of questions and corresponding rules and set of answers.
\end{abstract}

\section{Categories and Subject Descriptors}

D.2.2 [Software Engineering]: Design Tools and Techniques

\section{General Terms}

Management, Measurement, Documentation

\section{Keywords}

software product line engineering, decision support systems

\section{INTRODUCTION}

Currently an increasing number of companies aim to adopt a software product line engineering (SPLE) approach with the goal to enhance the quality of products, reduce timeto-market and optimize production costs. The benefits for adopting software product line engineering have been documented by several researchers and also mentioned in experience reports. In parallel, it is generally acknowledged

Permission to make digital or hard copies of all or part of this work for personal or classroom use is granted without fee provided that copies are not made or distributed for profit or commercial advantage and that copies bear this notice and the full citation on the first page. To copy otherwise, to republish, to post on servers or to redistribute to lists, requires prior specific permission and/or a fee.

SPLC 2013 workshops, Aug 26-30 2013, Tokyo, Japan

Copyright 2013 ACM 978-1-4503-2325-3/13/08 ...\$15.00. that the decision to adopt an SPLE approach needs to be performed carefully due to the different risks involved in taking such an important decision. To mitigate the potential risks of the transition to SPLE, several approaches have been proposed that include many different rules for analyzing the feasibility of the SPLE adoption and the selection of transition process $[1,2,3]$. Unfortunately, due to the many different rules and their dependencies it is not easy to apply these manually and likewise provide a proper decision with the corresponding justification. There are existing decision modeling approaches [4], for which the structure of decision making is similar to the approach proposed in this paper. However, the main difference is that existing decision modeling approaches for SPLE focus on handling the variability during the application of SPLE. This paper demonstrates the application to survey and select the proper transition strategy before applying SPLE.

In this paper, we propose the tool Transit-PL, a web based decision support system for analyzing the feasibility of SPLE for an organization and selecting the appropriate transition strategy. Transit-PL is based on a conceptual framework that we have developed after a thorough domain analysis on SPLE adoption studies from the existing literature. The conceptual framework represents the concepts that are needed in the decision making process for transitioning to SPLE. Transit-PL can be used to develop customized decision support system for selected strategies using different questions and the corresponding rules and set of answers. Besides the definition of questions, rules and answers the tool provides mechanisms to customize and generate reports including the corresponding justifications of the resulting decisions. The tool is freely available on the Web and can be used by organizations that aim to adopt SPLE.

The remainder of the paper is organized as follows. In section 2 we describe the conceptual framework on which the tool is based. Section 3 describes the tool architecture. Section 4 provides an example scenario for applying the tool. A final section concludes the paper.

\section{CONCEPTUAL FRAMEWORK}

Figure 1 shows the conceptual framework on which TransitPL is based. Transit-PL is used to provide a Decision on the SPLE Feasibility and selection of Transition Strategy. The decision is defined by a number of Steps that include asking a set of Questions and triggering Rules. Answers can trigger Rules, which define the score for the corresponding decisions. The scheduling of the questions and the rules is defined by Scheduler. Question can be a general question, 
feasibility question or strategy question. A general question aims to extract information about the company that does not impact the selection of the decision making process. SPLE feasibility questions aim to check whether it is feasible at all to transition to SPLE. Finally, the questions related to a strategy are to find the proper SPLE transition strategy. Some questions in the system can be related to both the feasibility of adopting SPLE and the related transition strategy. After the questioning process a report is generated by ReportGenerator.

Figure 1: Metamodel for decision making.

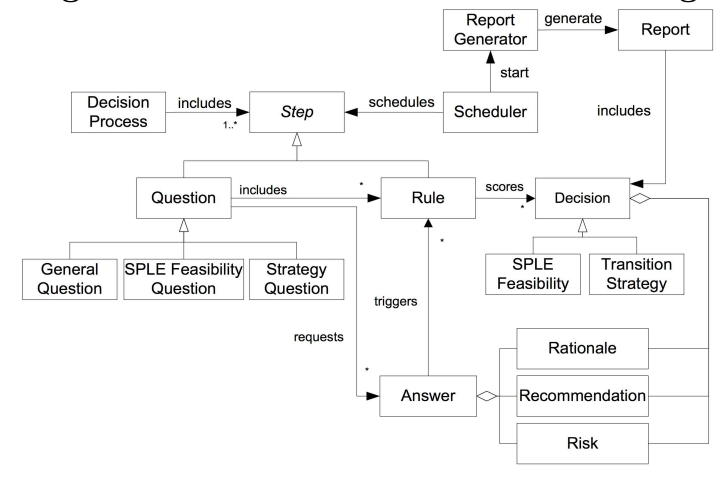

\section{TRANSIT-PL ARCHITECTURE}

Transit-PL ${ }^{1}$ has been implemented as a web-based tool and made freely available. The tool is developed using Ruby on the server-side and AngularJS ${ }^{2}$ on the client-side, and deployed to Heroku ${ }^{3}$ cloud application platform. The tool can be used both by decision support designer and decision makers. Decision support designers can use the toolset to define and configure a decision support process. Each decision support process can be stored (in JSON file format) and made publicly available in the tool, which enables a more rigorous validation throughout various runs and feedbacks. Decision makers can select and use the defined decision support systems to support the decision on the adoption of SPLE. In the following subsections we will explain each process in detail.

\subsection{Defining the Decision Support}

For defining a decision support process the necessary strategies, questions, answers and rules need to be identified. Typically this will require the analysis and extraction of information from knowledge sources that include the required content.

The first phase in the definition of the decision support is to select and define the SPLE transition strategies that will be used in the decision support system. Examples of these strategies are Incremental Introduction, Tactical Approach, Pilot Project, Big Bang Strategy. The next step in the definition process is the description of the questions and the rule set for each question associated with possible answers. The template for this is shown in Table 1.

As stated before a question may be a type of general, feasibility or strategy-related question. We further distinguish among the following questions based on the required format

\footnotetext{
$1_{\text {http: //transitpl.herokuapp.com }}$

2 http://www. angularjs.org

3 http://www.heroku.com
}

Table 1: Question template with a rule set.

\begin{tabular}{|l|l|}
\hline Element & Description \\
\hline ID & $\begin{array}{l}\text { Presents a unique identifier to distinguish } \\
\text { the different questions. }\end{array}$ \\
\hline Description & Short explanation of the question \\
\hline Question & $\begin{array}{l}\text { Defines whether the question is a gen- } \\
\text { eral question, SPLE feasibility question, or } \\
\text { strategy related question. }\end{array}$ \\
\hline Possible & $\begin{array}{l}\text { Describes the expected answers and the re- } \\
\text { quired format to the question. }\end{array}$ \\
\hline Rules & $\begin{array}{l}\text { Describes the set of rules that are triggered } \\
\text { by the provided answer. Each rule has } \\
<\text { Score }>\text { a numerical value for feasibility } \\
\text { or the predefined transition strategies that } \\
\text { will be considered. } \\
<\text { Rationale }>\text { the justification provided for } \\
\text { the corresponding answer. } \\
<\text { Risk }>\text { the possible risks explained for the } \\
\text { corresponding answer. } \\
<\text { Recommendation }>\text { the recommendation } \\
\text { provided for the corresponding answer. }\end{array}$ \\
\hline
\end{tabular}

of the answers: text-input, numeric-input, single-select and multiple-select. As the name suggests, text-input question gathers a piece of text from user and no more configuration is required. Numeric-input can be used to get a number. A minimum and maximum value limit for the answer should be specified for this type. Single-select refers to a question with one or more options for which only one can be selected. Multiple-select question is same as single-select except multiple options can be selected as the answer.

After the definition of questions with possible answers, rules are defined based on the answer set. Rules have conditions determining the action. These elements focus on the impact of an input, answers given to questions, on the transition process and generate helpful suggestions. Each rule is linked to SPLE feasibility or a transition strategy. A rule has the following structure: if $<$ condition $\rangle$ then $\langle$ actions $\rangle$, in which condition becomes true or false according to given answer that rule is applied to, and actions refer to a score, and a list of explanations providing reasoning and suggestions for supporting decision process. Score is between -1 and 1 indicating the impact of given answer in transition process. Table 2 lists corresponding conditions to each question type.

Table 2: Question types and corresponding rule conditions.

\begin{tabular}{|l|l|}
\hline Question Type & Rule Condition \\
\hline Text-input & has - keyword(word $)$ \\
\hline Numeric-input & in - range $($ lower, upper $)$ \\
\hline Single-select & in $-\operatorname{set}(S: S \subseteq A, A:$ set of answers $)$ \\
\hline Multiple-select & in $-\operatorname{set}(S: S \subseteq A, A:$ set of answers $)$ \\
\hline
\end{tabular}

has-keyword is a function to look for a word in a text. If the word passed to has-keyword function is found in the answer, then function returns true, otherwise false. in-range function becomes true if the answer to numeric-input question is in (lower, upper), otherwise false. in-set(S) function checks whether the given answer is a subset of set $S$, which can be any subset of all possible answers. The set $S$ can 
be described by combining elements in answer set $A$ with logical predicates. Table 3 gives the list of actions that can be defined within a rule and their explanations.

Table 3: List of rule actions.

\begin{tabular}{|l|l|}
\hline Rule Actions & Description \\
\hline Score & $\begin{array}{l}\text { A value between }-1 \text { and 1 indicating } \\
\text { the impact of given answer in transi- } \\
\text { tion process. }\end{array}$ \\
\hline Justification & The reasons behind this rule. \\
\hline $\begin{array}{l}\text { Potential } \\
\text { Risks }\end{array}$ & $\begin{array}{l}\text { The potential risks may be faced ac- } \\
\text { cording to given answer to the question. }\end{array}$ \\
\hline $\begin{array}{l}\text { Recommended } \\
\text { Step }\end{array}$ & $\begin{array}{l}\text { The step that is recommended to be } \\
\text { taken in the transition process. }\end{array}$ \\
\hline
\end{tabular}

\subsection{Using the Decision Support}

In defining the decision support phase, the decision maker uses the configured system and gets support for transition to SPLE via various strategies. Scheduler component of Transit-PL gathers the questions from the preconfigured knowledge base that has been defined before, and presents this to the decision maker to retrieve answers. Once the decision maker answers the questions, ReportGenerator component applies the rule set of each question to the corresponding answer. Each answer triggering one or more rules will result in a complete transition report explaining SPLE feasibility and applicability of transition strategies with rationales, risks, and recommended steps. In addition, the report provides the overall summary for the feasibility of SPLE and the selection of transition strategies with a pie chart and a histogram respectively.

The described decision support is based on overall scores for feasibility condition and a set of strategies. These scores are used to generate the charts as well to demonstrate the results. An overall score is the average of the scores that is defined by the rules of the questions. The scores are included in the average in case the corresponding condition section of the rules is triggered by the given answer. The answers that do not trigger any rule will not have an effect on the final score. In the current system we have adopted equal weight for the scores for the rules of a question.

\section{EXAMPLE SCENARIO}

In this section we illustrate the decision support system definition and usage process with Transit-PL for a particular scenario $^{4}$. The scenario consists of 35 questions, and more than 200 rules corresponding to these questions.

In the decision support definition process first the strategies are defined. Figure 2 shows the snapshot of the tool from which existing decision plans (decision support process) can be downloaded. Each plan is represented a title, whether the plan is publicly available or not, a link to the plan, and actions can be performed on the plan.

In case it is chosen to create a new decision support process then the subsequent three steps need to be followed. The first step is to give a name to the plan. The second step is to create transition strategies. A strategy consists of a name and description.

Figure 3 shows a list of strategies and a feasibility condition created for the demo plan.

\footnotetext{
$4_{\text {http: //transitpl.herokuapp.com/public/514100110bb0a50002000001 }}$
}

Figure 2: List of decision plans previously created.

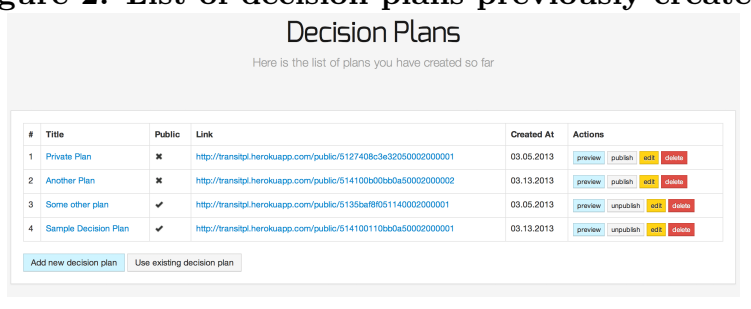

Figure 3: Defining transition strategies for the plan.

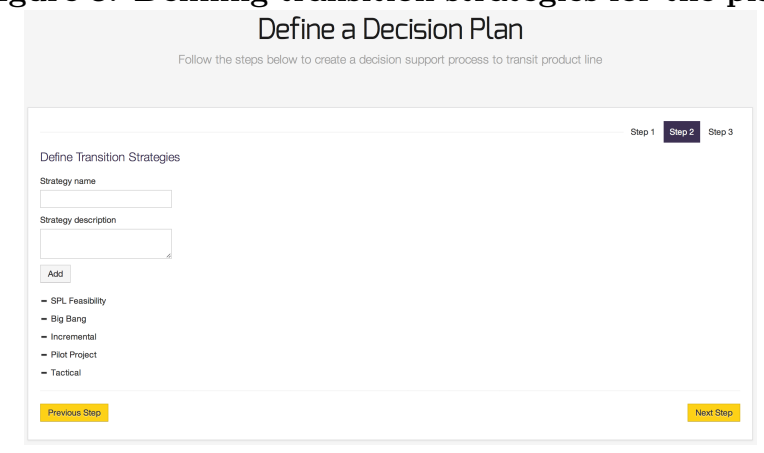

The final step is to create questions and configure rules using the possible answers for each question. Figure 4 demonstrates a question for the sample plan, which is a singleselect question expecting an option to be selected by the user. The question configuration part is followed with a preview showing the live demo for that question configuration as if it is presented to a user. Below a part for rules appears. This part starts with a textual description of the question and then each rule created for that question is listed. This whole question and rule description section corresponds to the template given in Table 1.

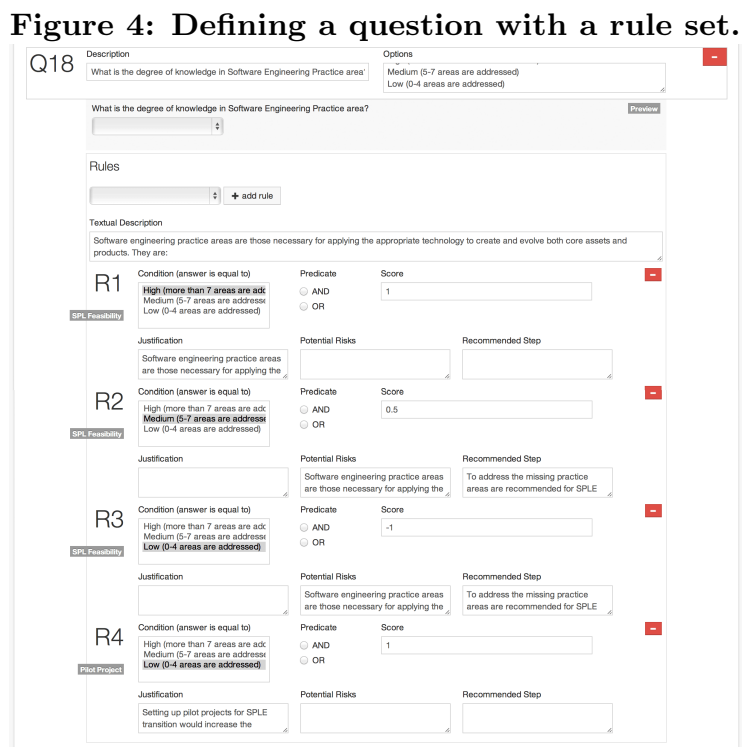

Figure 5 shows a list of questions to be answered for which only a small fraction of questions is given due to space limitation. These questions are expected to be defined previously 
through the decision support process definer and connected with a set of rules. In addition, the sections that are shown on the generated report can be configured using the checkboxes at the bottom. By this way, a decision maker can create customized reports by including or excluding different sections.

Figure 5: Preview of a decision plan.

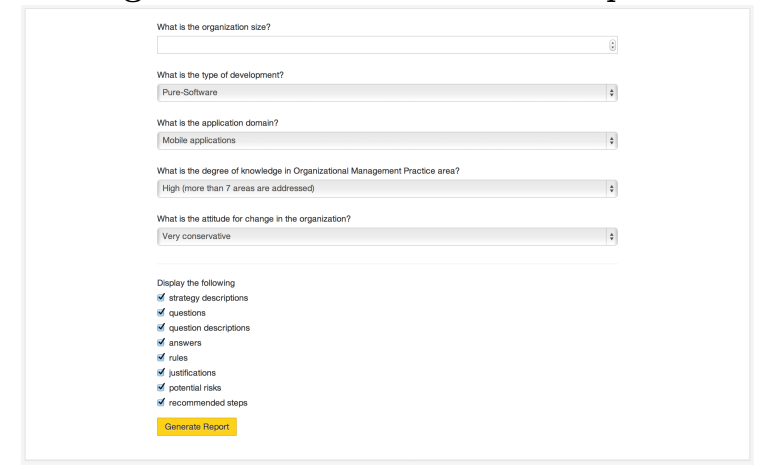

After a user or decision maker answering the questions, a report is generated. The report is formed by question descriptions, answers, and corresponding explanations for these answers. Figure 6 provides a sample view from the report. The report not only provides descriptions, but also conditions of rules that become true for the answer and related reasoning behind that rule. In this way, user is able to observe the effects of their current situation for adopting SPLE through these explanations.

Figure 6: Generated report section for a question.

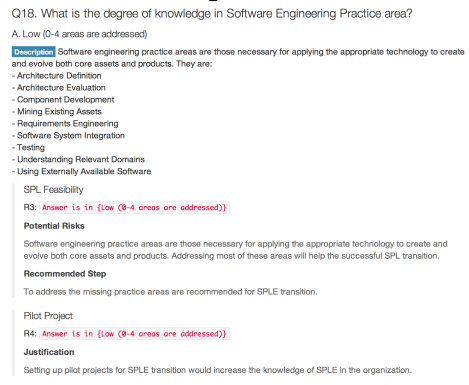

The textual section is followed by two charts for which a snapshot is given in Figure 7. The overall summary for SPLE feasibility condition is given with a pie chart, while the strategies are displayed through a histogram for comparison. The overall summaries are created via the final score for feasibility condition and transition strategies.

\section{CONCLUSION}

Transit-PL proposes a common skeleton for implementation of decision support process for adopting SPLE. The tool provides a decision support framework based on questions, rules and strategies with report generation capabilities to guide the transition process. The organizations that are planning to adopt SPLE can benefit from Transit-PL in the following ways:

- An initial contact point before transition process
Figure 7: Generated report section showing overall results.

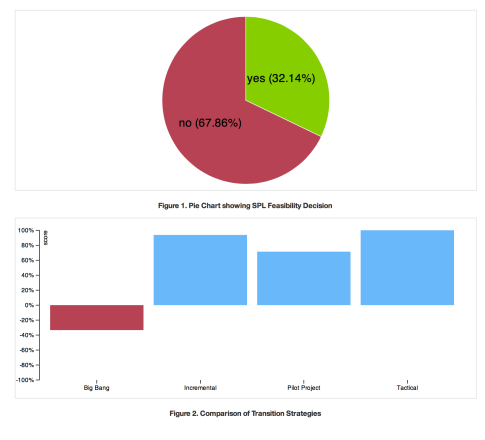

- Creation of decision plans and collaboration of different ideas via these plans

- Validation of plans by sharing with others and getting feedback

- A detailed report on feasibility of SPLE and applicability of transition strategies based on given answers

- Ability to (re)use and (re)design the predefined set of rules that are extracted from literature

We will improve Transit-PL by extending the notion of decision plan by decision flow for which the whole plan becomes a decision flow with subroutines, conditionals and blocks. We also plan to extend the question and rule set in the future.

\section{REFERENCES}

[1] C. W. Krueger. Easing the transition to software mass customization. In Revised Papers from the 4 th International Workshop on Software Product-Family Engineering, PFE '01, pages 282-293, London, UK, UK, 2002. Springer-Verlag.

[2] K. Pohl, G. Böckle, and F. Van Der Linden. Software product line engineering: foundations, principles, and techniques. Springer, 2005.

[3] K. Pohl, J. D. McGregor, L. M. Northrop, and S. Jarrad. Guest editors' introduction: Initiating software product lines. IEEE SOFTWARE, 19(4):0024-27, 2002.

[4] K. Schmid, R. Rabiser, and P. Grünbacher. A comparison of decision modeling approaches in product lines. In Proceedings of the 5th Workshop on Variability Modeling of Software-Intensive Systems, pages 119-126. ACM, 2011. 


\section{APPENDIX}

\section{A. PRESENTATION PLAN}

Transit-PL tool proposes a framework for decision support of adopting SPLE. Using the tool, a decision support designer can create a decision support process through the concepts defined in this paper. Plus, tool offers a predefined set of questions and rules, which we created through various resources. The presentation of tool will consist of the following steps:

\section{A.1 Short discussion on Decision Support Sys- tems (DSS) and their applicability in Soft- ware Engineering}

DSSs are computer-based information systems supporting business or organizational decision-making activities. These systems are used in various areas for help in making decisions.

\section{A.2 Short presentation on Software Product Line Engineering (SPLE) transition pro- cess and the existing strategies}

Transition to SPLE has been studied and various strategies are proposed. However, the application of these transition decision processes is currently manual. The idea of automating this cumbersome process is to be presented.

\section{A.3 Explanation of concepts behind Transit- PL}

Transit-PL is based on a metamodel that describes the decision support process. The concepts behind the tool consist of strategies, questions and rules. These concepts will be explained.

\section{A.4 Definition of decision support process with Transit-PL}

The design of a decision support process refers to a decision plan in Transit-PL. A plan is created and results are gathered in two steps: defining the decision support and using the decision support. Each step will be explained through a sample execution by defining questions, their possible answers, and configuration of rules for these answers. Once the sample plan is created, two case studies will be given over two different organizations. We will execute the sample process and examine the reports generated for these cases.

\section{A.5 Summary and possible improvements for the tool}

The organizations planning to adopt SPLE can benefit from Transit-PL in various ways. The benefits from the tool can be even further improved by extending the notion of decision support through a transition decision flow. In fact, the current question and rule set can be extended as well. The ideas for possible improvement will be discussed. 